\title{
Histoplasmosis pulmonar crónica en un adulto inmunocompetente
}

\author{
Chronic pulmonary histoplasmosis in \\ an immunocompetent adult
}

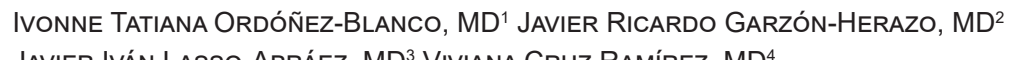

JAVIER IVÁN LASSO-APRÁEZ, MD³ VIVIANA CRUZ RAMÍREZ, MD ${ }^{4}$

\section{Resumen}

La histoplasmosis es la micosis endémica más frecuente a nivel mundial. Su aumento es proporcional con la creciente necesidad de terapias inmunosupresoras para el tratamiento de distintas enfermedades. Causada por el microorganismo Histoplasma capsulatum, la forma clínica más frecuente es la pulmonar, la cual se clasifica, según el tiempo de presentación, en aguda, subaguda y crónica. La histoplasmosis crónica representa el 8\% de los casos; esta se caracteriza por la formación progresiva de cavitaciones y extensa destrucción del parénquima pulmonar. Comúnmente se describe en pacientes con enfermedad pulmonar crónica y antecedente de tabaquismo. En Colombia hasta el 10\% de los casos no se asocia con factores de riesgo. Dada su importancia, y la escasa información reportada en la literatura, presentamos el caso de un hombre de 50 años atendido en el Hospital Universitario San Ignacio; a quien se diagnosticó histoplasmosis pulmonar crónica con estudios de inmunodeficiencia normales, identificándose como único factor de riesgo su ocupación como demoledor de construcciones.

Palabras clave: histoplasmosis pulmonar crónica.

\begin{abstract}
Histoplasmosis is the most common endemic mycosis around the world, increasing proportionally with the growing need of immunosuppressive therapies for the treatment of different diseases. Caused by the microorganism Histoplasma capsulatum, the most frequent clinical form is the pulmonary one, which is classified, according to the time of presentation, in acute, subacute, and chronic. Chronic histoplasmosis accounts for $8 \%$ of cases, characterized by the progressive formation of cavities and extensive destruction of the pulmonary parenchyma, typically described in patients with chronic pulmonary disease and smoking history. In Colombia, up to $10 \%$ of cases is not associated with risk factors. Given its importance and the scarce information reported in the literature, we present the case of a 50-year-old man treated at Hospital Universitario San Ignacio who was diagnosed with chronic pulmonary histoplasmosis and in whom immunodeficiency studies were normal, identifying themselves as unique factor of risk its occupation as demolisher of constructions.
\end{abstract}

Keywords: Chronic pulmonary histoplasmosis.

\footnotetext{
${ }^{1}$ Hospital Universitario San Ignacio, Departamento de Medicina Interna. Bogotá, Colombia.

${ }^{2}$ Hospital Universitario San Ignacio, Unidad de Infectología. Bogotá, Colombia.

${ }^{3}$ Hospital Universitario San Ignacio, Unidad de Neumología. Bogotá, Colombia.

${ }^{4}$ Pontificia Universidad Javeriana, Departamento de Epidemiología. Bogotá, Colombia.

Autor de correspondencia

Javier Ricardo Garzón Herazo. Ak. 7 \#19141. Tel.: +57 (310)338.13.76. Correo electrónico: garzonjavier@yahoo.com.

Recibido: 21/08/17. Aceptado: 10/11/17.
} 


\section{Introducción}

La histoplasmosis es la micosis endémica más frecuente a nivel mundial, con una tasa anual de infección estimada de 1\% (1). La infección constituye un desbalance entre el tamaño del inóculo y la capacidad del sistema inmune para contrarrestarla. En nuestro país, la infección por virus de la inmunodeficiencia humana (VIH) se describe como el principal predisponente para el desarrollo de histoplasmosis; sin embargo, hasta en $20 \%$ de los casos no se logra identificar ningún factor de riesgo (2).

La infección por histoplasma puede ser pulmonar, mediastinal, del sistema nervioso central, articular, vascular o por infección diseminada. A su vez, según el tiempo de presentación, las formas pulmonares se clasifican en histoplasmosis pulmonar aguda, subaguda o crónica. Los hallazgos radiológicos más frecuentes son las opacidades en parches (en la histoplasmosis pulmonar aguda), micronódulos y nódulos (en la histoplasmosis diseminada), cavitaciones (en la forma crónica), adenitis y fibrosis (en las formas mediastinales), histoplasmoma y broncolitiasis (1).

La histoplasmosis pulmonar crónica, descrita en $8 \%$ de los casos, se presenta con la formación de cavitaciones y destrucción parenquimatosa, tiene un curso de instauración progresivo y típicamente se describe en pacientes con enfermedad pulmonar crónica establecida de cualquier causa y antecedente de tabaquismo $(3,4)$.

Presentamos el caso de un hombre de 50 años atendido en el Hospital Universitario San Ignacio a quien se diagnosticó histoplasmosis pulmonar crónica. Los estudios para inmunodeficiencia fueron normales, identificándose como único factor de riesgo su ocupación como demoledor de construcciones.

\section{Descripción del caso}

Se trata de un paciente de 50 años, albañil y con cuadro clínico de 12 meses de evolución caracterizado por tos seca, ocasionalmente expectoración amarilla o hemoptoica, disnea grado 3 en la escala mMRC ( $m o-$ dified Medical Research Council), diaforesis nocturna y pérdida de peso de $13 \mathrm{~kg}$. Como antecedentes tuvo hipertensión arterial en tratamiento con antagonista de calcio y no fue fumador.

En el examen físico, su estado general se encontró regular, con presión arterial de 150/95 $\mathrm{mm} \mathrm{Hg}$, frecuencia cardíaca de $137 \mathrm{lpm}$, frecuencia respiratoria de $26 \mathrm{rpm}$, temperatura de $37^{\circ} \mathrm{C}$ y saturación de oxígeno de $70 \%$ al aire ambiente. Uso de músculos accesorios de la respiración y estertores inspiratorios bilaterales; sin adenopatías, visceromegalias o lesiones en piel o mucosas.

Hemograma con 14390 leucocitos $/ \mathrm{mm}^{3}, 12400$ neutrófilos $/ \mathrm{mm}^{3}, 1180$ linfocitos $/ \mathrm{mm}^{3}, 700$ monocitos/ $\mathrm{mm}^{3}, 14 \mathrm{~g} / \mathrm{dL}$ de hemoglobina, $46 \%$ de hematocrito, 290200 plaquetas $/ \mathrm{mm}^{3}$, velocidad de sedimentación globular $80 \mathrm{~mm} / \mathrm{h}$. Las pruebas de función renal y hepática fueron normales. Gases arteriales con hipoxemia severa y equilibrio ácido básico $\left(\mathrm{pH} 7,42 ; \mathrm{PCO}_{2}\right.$ $32,2 \mathrm{~mm} \mathrm{Hg} ; \mathrm{PO}_{2} 39 \mathrm{~mm} \mathrm{Hg} ; \mathrm{HCO}_{3} 20,6 \mathrm{mmol} / \mathrm{L}$; $\mathrm{BEb}-3,1 \mathrm{mmol} / \mathrm{L} ; \mathrm{SO}_{2} 75,2 \%$ ). Hemocultivos negativos a los 5 días de incubación.

Baciloscopia seriada negativa y proteína C-reactiva (PCR) MTB-RIF (GeneXpert) negativa. La radiografía de tórax mostró engrosamiento de paredes bronquiales con opacidades en vidrio esmerilado y focos de consolidación bilaterales interpretados como bronquiectasias, iniciándose tratamiento con cefepima. La tomografía de tórax con medio de contraste demostró hipertensión pulmonar (tronco de la arteria pulmonar de $31 \mathrm{~mm}$ ); adenomegalias paratraqueales, subcarinales y en la ventana aortopulmonar; bronquiectasias cilíndricas y saculares, vidrio esmerilado y nódulos con lesiones cavitadas de pared gruesa, peribronquiales, distribuidos en los lóbulos superiores, inferiores y medio (Figura 1 y 2).

Con los hallazgos tomográficos y la impresión clínica de infección oportunista se hizo fibrobroncoscopia diagnóstica, encontrándose antracosis, eritema y pus en árbol bronquial. Se tomaron muestras con lavado broncoalveolar en el que las coloraciones de Gomori y PAS demostraron estructuras fúngicas de morfología compatible con histoplasma (Figura 3). Se realizó diagnóstico de histoplasmosis pulmonar crónica y se inició tratamiento con anfotericina B. 


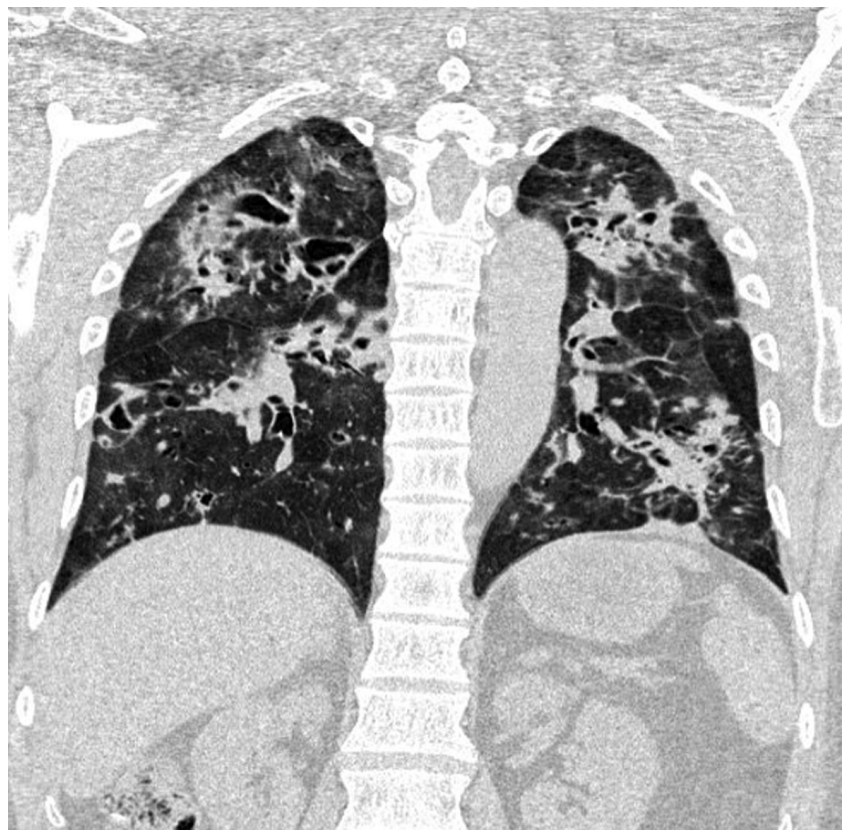

Figura 1. Tomografía axial computarizada (TAC) coronal de tórax. Adenomegalias paratraqueales, en ventana aortopulmonar y subcarinales. Pulmones ligeramente disminuidos de tamaño. Bronquiectasias cilíndricas y saculares. Parches de vidrio esmerilado en lóbulos superiores, medio e inferiores.

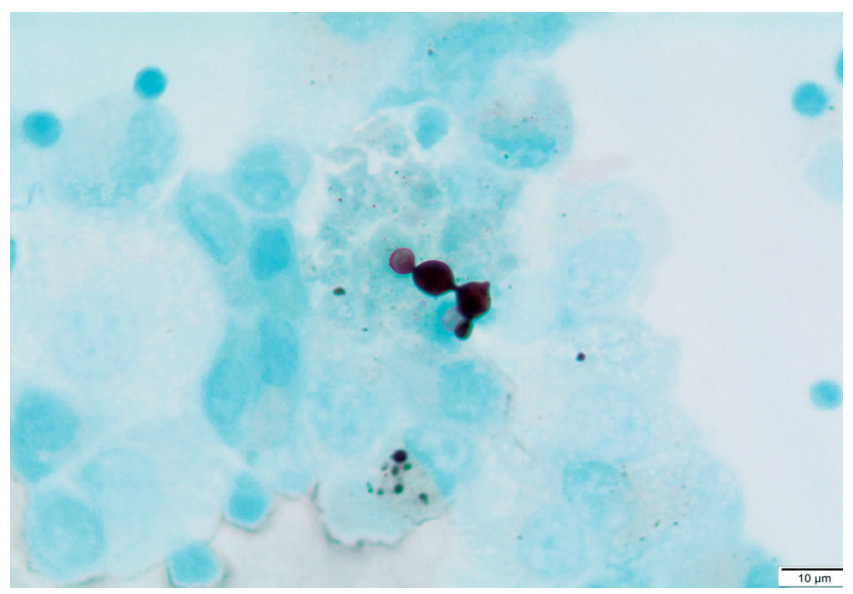

Figura 3. Coloración de Gomori. Histoplasma.

Se amplían estudios para inmunosupresión con ELISA-VIH no reactivo, inmunoglobulinas IgA, IgM, IgE e IgG levemente elevadas y citometría de flujo con 399 linfocitos $\mathrm{T} \mathrm{CD}_{4}^{+} / \mathrm{mm}^{3}(41,7 \%)$ que descartó inmunodeficiencia primaria. El único factor de riesgo relacionado fue su ocupación laboral en construcción y demolición de edificios antiguos.

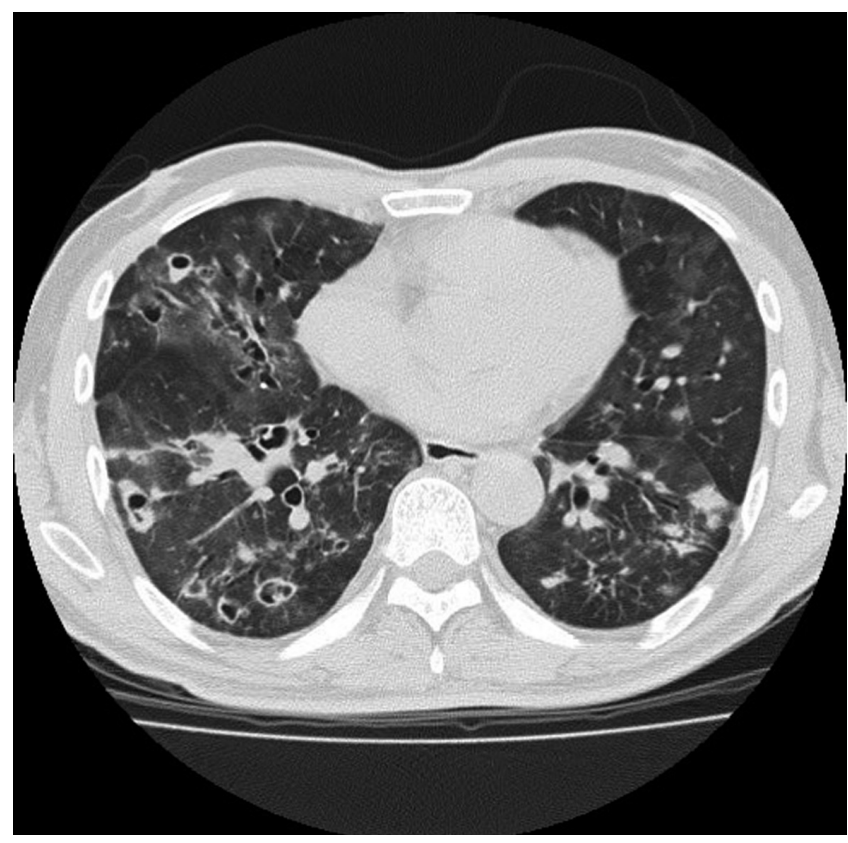

Figura 2. TAC de tórax. Múltiples nódulos y lesiones cavitadas de pared gruesa, de localización peribronquial en lóbulos superiores, lóbulo medio y lóbulos inferiores.

Después de 14 días de tratamiento con anfotericina B hubo mejoría clínica significativa y se dio de alta del hospital con prescripción de itraconazol $200 \mathrm{mg} /$ día por vía oral con plan de 6 o 12 meses de acuerdo con su evolución.

El paciente asistió a control 3 meses después de su egreso y refirió mejoría satisfactoria de la disnea y resolución de la tos. Se continuó con el mismo tratamiento, y 8 meses después en el control tomográfico hubo mejoría notoria de las lesiones pulmonares, completándose 12 meses de tratamiento (Figura 4 y 5).

\section{Discusión}

La histoplasmosis se ha descrito como la micosis endémica más frecuente en América anglosajona y países latinoamericanos, con una incidencia de 0,4 casos por 100000 habitantes $(2,5)$. Se estima que, en Estados Unidos cerca de 30 millones de personas padecen la infección (6). Dentro de los factores de riesgo se encuentran la infección por VIH, como el más importante, también se describe el uso prolongado de esteroides, neoplasias, trasplante de órgano sólido y de mé- 


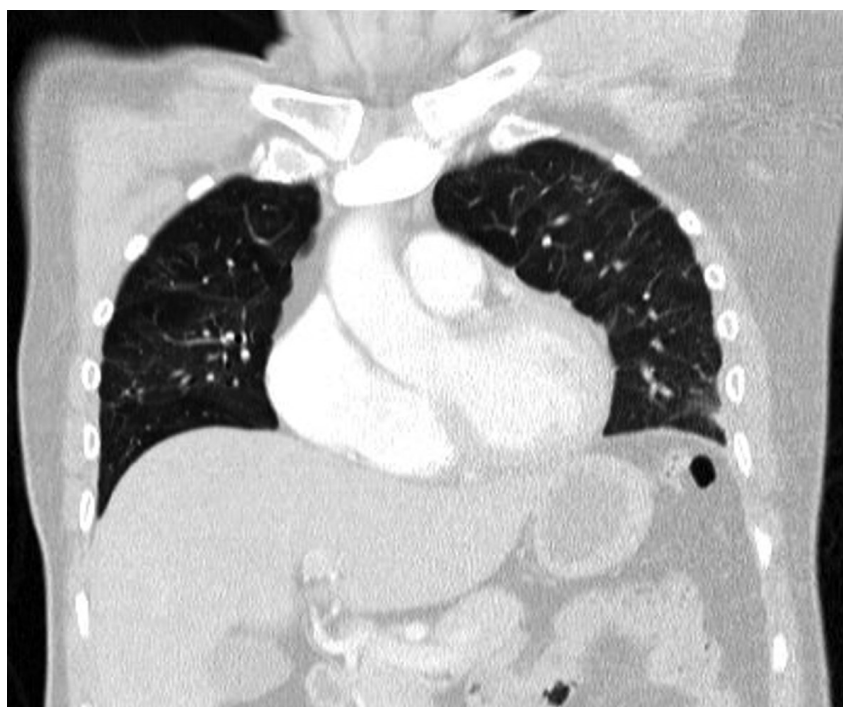

Figura 4. TAC coronal de tórax control. Nódulos con densidad de vidrio esmerilado y algunos con densidad de tejidos blandos de localización periférica, especialmente en lóbulos inferiores con diámetros hasta de $7 \mathrm{~mm}$.

dula ósea, cirrosis y alcoholismo $(5,6)$. Asimismo, se han descrito casos de histoplasmosis pulmonar crónica en pacientes con enfermedad de Crohn o enfermedad reumatológica, quienes deben usar medicamentos inhibidores del factor de necrosis tumoral (antiTNF) $\alpha(7)$.

El número de casos de histoplasmosis reportados en pacientes sin antecedentes patológicos que habitan en zonas endémicas ha incrementado. En nuestro país hasta en $10 \%$ de los casos no se encuentra asociación causal con ningún factor de riesgo, y a nivel mundial hasta en un $20 \%$ (2). El desarrollo de la infección en pacientes inmunocompetentes se relaciona con la exposición a un inóculo grande, favoreciéndose la multiplicación de las esporas dentro del parénquima pulmonar (6).

$\mathrm{Su}$ principal causante es la especie Histoplasma capsulatum, adquirida por la inhalación de esporas o fragmentos de micelios durante la remoción del suelo donde se encuentra el hongo $(1,4)$. Los microinóculos del hongo se hallan en suelos ricos en estiércol de aves o guano, árboles, edificaciones viejas o en cuevas de murciélagos, siendo la población más afectada aquella dedicada a labores en estos ambientes, tales como leñadores, albañiles y espeleólogos (1). Una vez inhalado, y de acuerdo con la competencia inmunológica del

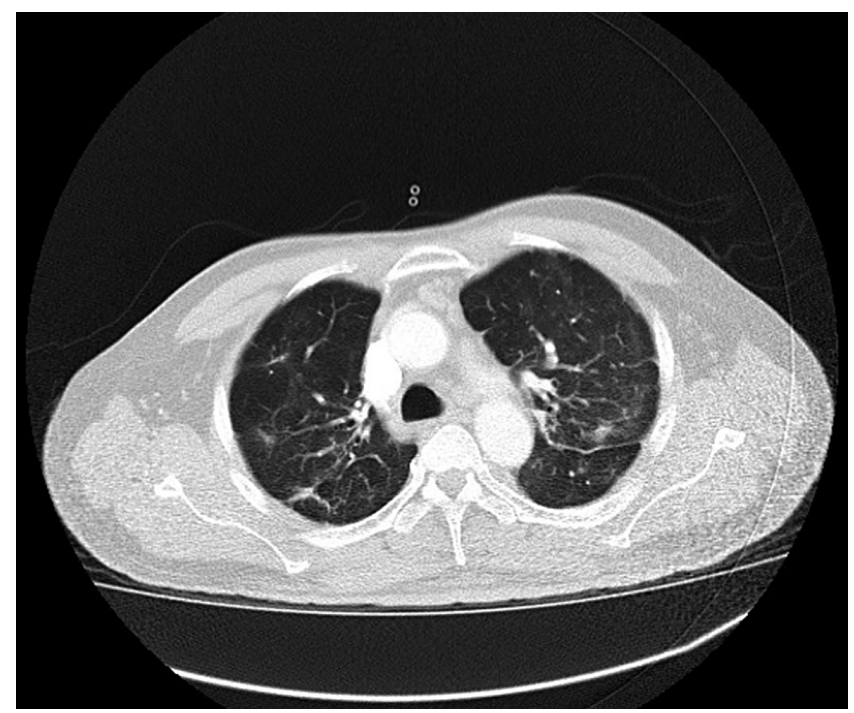

Figura 5. TAC de tórax control. Aumento en el número de ganglios prevasculares y paratraqueales sin configurar adenomegalias. Tractos de fibrosis y bronquiectasias por tracción en lóbulos superiores, principalmente derecho.

huésped, supera las barreras mecánicas e inmunes de la vía respiratoria e inicia un proceso inflamatorio en el parénquima pulmonar que, de no controlarse, conlleva a diseminación sistémica vía hematógena. En este caso no había antecedentes médicos patológicos, uso de terapia inmunosupresora, infección por VIH, linfopenia $\mathrm{T} \mathrm{CD}_{4}^{+}$idiopática o síndrome de hiperIgE; no obstante, el antecedente ocupacional se consideró como claro factor de riesgo (8).

El $90 \%$ de las infecciones agudas es subclínica, los demás pacientes tienen un cuadro clínico similar al de la influenza, algunos de estos con neumonitis en parches que se resuelve espontáneamente la mayoría de las veces, quedando con focos de infección latentes que se pueden reactivar si la inmunidad del huésped decae, dando lugar a una infección diseminada. Las formas crónicas usualmente se describen en hombres mayores de 50 años con enfermedad pulmonar previa (principalmente enfisema) y fumadores $(7,8)$.

La primera descripción de histoplasmosis pulmonar crónica en un paciente vivo se hizo en 1948 (9). Esta constituye hasta el $39 \%$ de los casos, tiene un curso lento y progresivo, y el compromiso pulmonar puede llevar incluso a grados variables de fibrosis. El ante- 
cedente de enfermedad pulmonar obstructiva crónica (EPOC) y tabaquismo es común en la mayoría de las series de casos reportados, el cual, junto con la exposición sostenida a grandes inóculos, contribuye a su desarrollo (6).

Esta forma de histoplasmosis se relaciona con enfermedad parenquimatosa previa, y característicamente se describe como una enfermedad cavitaria de los ápices pulmonares que muchas veces se confunde con la tuberculosis, retardando el diagnóstico de histoplasmosis pulmonar crónica $(6,7)$.

Las manifestaciones clínicas incluyen tos con expectoración purulenta, disnea, anorexia, pérdida de peso, astenia, adinamia y, ocasionalmente, fiebre (7). Los principales hallazgos radiológicos son enfisema con bulas de distribución heterogénea, retracciones hiliares y engrosamiento pleural, así como el desarrollo de nódulos. En etapas más tardías hay necrosis del parénquima, compromiso vascular y fibrosis $(10,11)$. Sin embargo, descripciones recientes en la literatura sugieren que las características definidas como típicas de la enfermedad tal vez no sean tan frecuentes.

En el año 2007, Kennedy y colaboradores describieron una serie de 46 pacientes afectados con histoplasmosis crónica durante un período de 24 años (desde 1976 hasta 2000). Se escogieron aquellos pacientes con síntomas y hallazgos radiológicos compatibles con histoplasmosis pulmonar por más de 6 semanas con cultivo o serología positiva (antígeno) (4).

Hubo una discreta predominancia de pacientes de género masculino afectados (52\%). En general, las características clínicas, radiológicas y la evolución fueron similares en los dos géneros. Solo el $28 \%$ de los pacientes era fumador activo, y el $41 \%$ tenía historia de tabaquismo. Hasta el $37 \%$ de los pacientes reportó un antecedente exposicional claro a un inóculo de histoplasmosis, entre ellos, excrementos de murciélagos y aves, sitios de construcción o demolición, o entornos agrícolas. Contrario a lo previamente descrito, la asociación con el grupo de pacientes con EPOC solo se encontró en $20 \%$ de los casos. En solo 13 pacientes se logró el aislamiento en medios de cultivo, mientras que en casi la totalidad se documentó títulos elevados del antígeno de histoplasma $(2,4)$.
La enfermedad cavitaria radiológica solo se evidenció en $30 \%$ de los pacientes en la tomografía de tórax. Por el contrario, la presencia de nódulos fue el principal hallazgo radiológico evidenciado (93\%), seguido de linfadenopatías (67\%), enfermedad cavitaria (30\%), enfisema (23\%), bronquiectasias (7\%) y fístula broncopleural (3\%). El compromiso pulmonar de nuestro paciente estuvo dado por una gran cantidad de bronquiectasias cilíndricas, así como la presencia de múltiples nódulos y lesiones cavitadas que mejoraron considerablemente en controles radiológicos después de tratamiento antifúngico.

El pequeño grupo de pacientes con enfermedad cavitaria tuvo mayor asociación con tabaquismo (directamente proporcional al índice tabáquico) y el antecedente de EPOC (87\% de los casos). En la mayoría de estos pacientes no se logró aislamiento a través de cultivo. Como se mencionó previamente, estos hallazgos quizás no deberían sorprendernos, teniendo en cuenta que las descripciones iniciales de la enfermedad se realizaron en sanatorios de pacientes con sospecha de tuberculosis. De acuerdo con los casos descritos en esta serie, la enfermedad cavitaria parece que representa solo un espectro de la histoplasmosis pulmonar crónica e infrecuentemente relacionada con los factores de riesgo clásicamente descritos de tabaquismo, EPOC u otra alteración parenquimatosa pulmonar (4).

El diagnóstico se hace con el aislamiento de H. capsulatum en cultivo, con una positividad en muestras pulmonares hasta del $60 \% \mathrm{y}$, aunque el antígeno urinario puede ser una herramienta, su principal utilidad es el diagnóstico de las formas diseminadas. Otros métodos de diagnóstico empleados son la medición de títulos de anticuerpos mediante fijación del complemento con sensibilidad variable de $73 \%$ a $95 \%$, con títulos superiores a 1:32 indicativos de enfermedad activa, y la inmunodifusión de bandas de precipitinas, con un rendimiento diagnóstico inferior (sensibilidad de 55\%) $(3,12)$.

En nuestro caso se llegó al diagnóstico a través del estudio en muestra de lavado broncoalveolar, en el cual se vieron las estructuras fúngicas. El diagnóstico histopatológico en tejido demuestra granulomas con necrosis de caseificación y, en su interior, levaduras y las cápsulas de hongos muertos (6). 
La piedra angular del tratamiento es el uso de itraconazol $600 \mathrm{mg}$ /día durante 3 días, seguido de 200-400 $\mathrm{mg} /$ día durante 18 a 24 meses. Ante la progresión de síntomas durante el tratamiento o la recidiva del cuadro se propone como alternativa el uso de anfotericina liposomal $(13,14)$.

El tratamiento de la histoplasmosis pulmonar crónica es largo y requiere un seguimiento periódico ante la posibilidad de recaídas en más del 15 de los pacientes $(2,14,15)$. En nuestro caso, en los controles realizados a los 6 y 12 meses del cuadro pulmonar, la evolución clínica del paciente continúa siendo favorable, con notable mejoría radiográfica y clínica.

\section{Conclusiones}

La histoplasmosis pulmonar crónica representa un reto médico en nuestro país, puesto que sus manifestaciones clínicas se asemejan a la tuberculosis pulmonar, siendo esta última de mayor prevalencia en Colombia. El caso presentado resalta la importancia de reconocer otras presentaciones radiológicas distintas a la clásicamente descrita, que podrían ser incluso más frecuentes. Se debe tener un alto índice de sospecha en pacientes con síntomas pulmonares crónicos asociados con cavitaciones, nódulos y bronquiectasias, incluso en ausencia de factores de riesgo descritos, como EPOC y tabaquismo, cada vez menos reconocidos como asociados con la condición. El tratamiento antifúngico por tiempo prolongado es altamente efectivo y mejora gran parte del compromiso parenquimatoso severo.

\section{Agradecimientos}

Liliana Arias, MD. Servicio de Radiología del Hospital Universitario San Ignacio.

Luis Fernando Jaramillo, MD. Servicio de Patología del Hospital Universitario San Ignacio.

\section{Responsabilidades éticas}

Protección de personas y animales: los autores declaran que para esta investigación no se han realizado experimentos en seres humanos ni en animales.
Confidencialidad de los datos: los autores declaran que han seguido los protocolos de su centro de trabajo sobre la publicación de datos de pacientes.

Derecho a la privacidad y consentimiento informa$d o$ : los autores obtuvieron el consentimiento informado de los pacientes y/o sujetos referidos en el artículo.

Este documento obra en poder del autor de correspondencia.

\section{Conflicto de intereses}

Los autores declaramos que no tenemos conflictos de intereses en la publicación de este trabajo y hemos seguido los protocolos establecidos en nuestra institución para la publicación de este material.

\section{Referencias}

1. Wheat LJ, Azar MM, Bahr NC, Spec A, Relich RF, Hage C. Histoplasmosis. Infect Dis Clin North Am. 2016;30(1):20727. DOI: $10.1016 /$ j.idc.2015.10.009.

2. Arango M, Castañeda E, Agudelo CI, De Bedout C, Agudelo CA, Tobón A, et al. Histoplasmosis: results of the Colombian national survey, 1992-2008. Biomed. 2011;31(3):344-56. DOI: $10.1590 / \mathrm{S} 0120-41572011000300007$.

3. Goodwin RA, Owens FT, Snell JD, Hubbard WW, Buchanan RD, Terry RT, et al. Chronic pulmonary histoplasmosis. Medicine (Baltimore). 1976;55(6):413-52.

4. Kennedy CC, Limper AH. Redefining the clinical spectrum of chronic pulmonary histoplasmosis: a retrospective case series of 46 patients. Medicine (Baltimore). 2007;86(4):252-8. DOI: 10.1097/MD.0b013e318144b1d9.

5. Hage CA, Azar MM, Bahr N, Loyd J, Wheat LJ. Histoplasmosis: up-to-date evidence-based approach to diagnosis and management. Semin Respir Crit Care Med. 2015;36(5):72945. DOI: $10.1055 / \mathrm{s}-0035-1562899$.

6. Pometta R, Trovato C, Viviani MA, Masini T, Conte D. Chronic pulmonary histoplasmosis in a patient with a recent history of tuberculosis and persistent round lung lesions. Eur J Clin Microbiol Infect Dis. 1999;18(3):229-31. DOI: https:// doi.org/10.1007/s100960050267.

7. Zöllner MSA, Rezende KMPEC, Birman S, Elias CPF, Arisawa EÂL, Santos MABDV. Clinical and evolutionary characteristics of four patients with pulmonary histoplasmosis reported in the Paraíba Paulista Valley region. Rev Soc Bras Med Trop. 2010;43(5):599-601. DOI: http://dx.doi. org/10.1590/S0037-86822010000500028. 
8. Santos JW, Michel GT, Lazzarotto M, Figaro JK, Spilmann D, Homrich GK. Chronic cavitary pulmonary histoplasmosis. J Bras Pneumol. 2009;35(11):1161-4. DOI: http://dx.doi. org/10.1590/S1806-37132009001100016.

9. Johnson HE, Batson R. Benign pulmonary histoplasmosis. Dis Chest. 1948;14(4):517-24. DOI: https://doi.org/10.1378/ chest.14.4.517.

10. García-Marrón M, García-García JM, Pajín-Collada M, Alvarez-Navascués F, Martínez-Muñiz MA, Sánchez-Antuña AA. Chronic pulmonary histoplasmosis diagnosed in a nonimmunosuppressed patient 10 years after returning from an endemic area. Arch Bronconeumol. 2008;44(10):567-70. DOI: $10.1157 / 13126837$.

11. Capone D, Wanke B, Monteiro PC, Lazéra MS, de Noronha Andrade G, do Valle AC, et al. Chronic pulmonary histoplasmosis in the State of Rio de Janeiro, Brazil. Mycopathologia. 1999;145(2):75-9. DOI: https://doi. org/10.1023/A:1007016414833.
12. Richer SM, Smedema ML, Durkin MM, Herman KM, Hage CA, Fuller D, et al. Improved diagnosis of acute pulmonary histoplasmosis by combining antigen and antibody detection. Clin Infect Dis. 2016;62(7):896-902. DOI: 10.1093/cid/ ciw007.

13. Lionakis MS, Holland SM. Human invasive mycoses: immunogenetics on the rise. J Infect Dis. 2015;211(8):1205-7. DOI: 10.1093/infdis/jiu411.

14. Wheat LJ, Freifeld AG, Kleiman MB, Baddley JW, McKinsey DS, Loyd JE, et al. Clinical practice guidelines for the management of patients with histoplasmosis: 2007 update by the Infectious Diseases Society of America. Clin Infect Dis. 2007;45(7):807-25. DOI: 10.1086/521259.

15. Pugsley HE, Brown AS, Cheung OT. Chronic cavitary histoplasmosis of the lung. Can Med Assoc J. 1963;88(13):646-9. 